\title{
Ontologies in Various Domains: A New Perspective of Ideas
}

\section{Tanaya Das*}

Computer Science and Engineering, Adamas University, India

*Corresponding Author: Tanaya Das, Adamas University, Kolkata, West Bengal, India.

Received: January 14, 2022; Published: January 22, 2022

\section{Introduction}

The term ontology came from two Greek words i.e., "Onto" and "logos". It denotes "study or knowledge about everything that actually exists". Philosophers defined the word "Ontology" in their own way. It is discipline of philosophy [1] that deals with structures of things, events, processes, and relations in every aspect of reality. In recent years, research in multidisciplinary fields has exploded in various sectors or domain. "Ontology" term from philosophy was adapted and repurposed to fit the needs of sectors or domain. Thecore goal of ontologies is to provide a vision of a domain. Ontologies consist of all relevant domain-specific terminologies and connections that help to identify core concepts and accumulate critical information related to the domain. Nowadays, ontology is deployed in various fields to capture the domain knowledge. The use of ontology in various sectors is shown in Fig.1 and explained further.

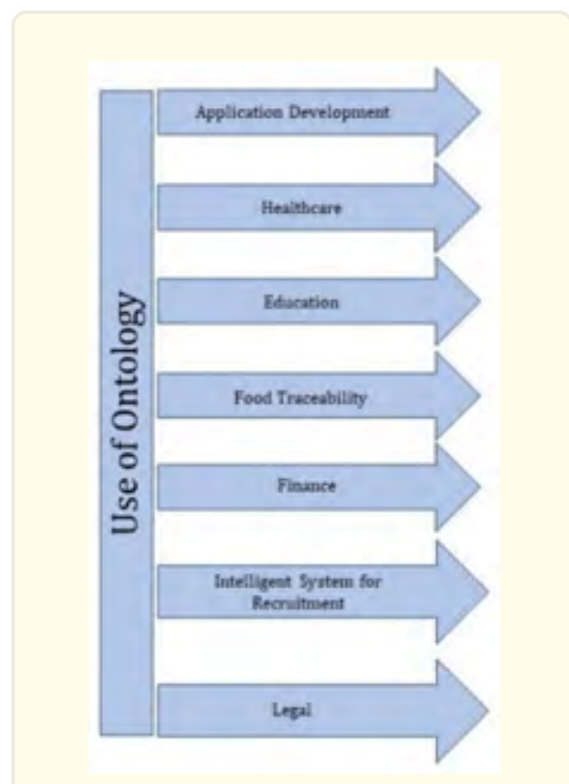

Figure 1: Ontology in various sectors.

\section{Ontology for Application Development}

An overview of various applications sectors [2] where ontology along with their usefulness have potential to deal with real issues. These sectors are knowledge management, semantic web, grid computing, multimedia and various pervasive computing environments. 


\section{Ontology for Food Traceability}

Nowadays, food ontology [3] are created to serve the purpose of food traceability. Its major purpose is to bring together the most representatives food concepts in a supply chain and trace their service items, processes, food, etc.

\section{Ontology for Healthcare}

In recent decades, advancement in medical treatment and information technologies have broadened the scope of traditional medical practices. It opened new possibilities for speedy, diversified and dynamic enterprise level applications. Healthcare domain ontology helps to build these applications at the time of need. Domain ontology for healthcare [4] discussed and handled challenges particularly during an emergency.

\section{Ontology for Intelligent system for Recruitment}

In today's era, Employment is one of the major areas that require attention. Few available technologies provide simplifying job searches. The study discussed in [5] shows creation of an intelligent website that would function as a service provider for recruiters. In this study the recruitment domain is shown in ontological form that provides some matching of searches between candidate resume and job offers.

\section{Ontology for Education}

Ontology for education domain resolves many issues of designing courses. Using ontology, minute details of various courses can be precisely represented. These course ontologies [6] can be given to students to choose their future courses based on their qualifications.

\section{Ontology for Legal}

Legal ontologies are useful to create legal knowledge bases. Legal ontologies play a key role in representing and processing legal information. legal ontologies [7] used by practitioners are required for major purposes like organizing and structuring information present in legal documents, reasoning and problem solving, searching of the legal content, and understanding the domain knowledge.

\section{Future Scope}

As explained above, ontologies are being used in various domains for many purposes. It can be used to understand some issues and structure those issues to find real solutions. It plays a key role in developing knowledge bases. Still, ontologies in various sectors are somewhat unexplored territory. Solutions are there, but there is no clear insight regarding how the ontologies can be used. When should we use ontologies, instead of databases? These questions enumerate several future research directions for preparing ontologies in domains like Pharmacy, Transport, Justice Delivery, etc.

\section{References}

1. Jacquette Dale. O.ntology. Acumen (2003).

2. Gaitanou P. “Ontologies and Ontology-Based Applications”. Metadata and Semantics, Springer, Boston, MA (2009): 289-298.

3. Pizzuti Teresa., et al. "Food Track \& Trace Ontology for Helping the Food Traceability Control”. Journal of Food Engineering 120 (2014): 17-30.

4. Zeshan Furkh and Radziah Mohamad. "Medical Ontology in the Dynamic Healthcare Environment". Procedia Computer Science10 (2012): 340-348.

5. García-Sánchez Francisco., et al. “An Ontology-Based Intelligent System for Recruitment”. Expert Systems with Applications 31.2 (2006): 248-263.

6. Ameen Ayesha., et al. "Creation of Ontology in Education Domain". 2012 IEEE Fourth International Conference on Technology for Education (2012). 
7. Ferrario Roberta., et al. "Towards an Ontological Foundation for Services Science: The Legal Perspective”. Approaches to Legal Ontologies (2010): 235-258.

Volume 2 Issue 2 February 2022

(C) All rights are reserved by Tanaya Das. 